\title{
Deuterium Fractionation and Ionization Degree in Massive Protostellar/cluster Cores
}

\author{
Huei-Ru Chen ${ }^{1}$, Sheng-Yuan Liu ${ }^{2}$, and Yu-Nung Su${ }^{2}$ \\ ${ }^{1}$ Institute of Astronomy \& Department of Physics, National Tsing Hua University, \\ Hsinchu 30013, Taiwan \\ email: hchen@phys.nthu.edu.tw \\ ${ }^{2}$ Institute of Astronomy \& Astrophysics, Academia Sinica, Taipei 10617, Taiwan \\ email: syliu@asiaa.sinica.edu.tw, ynsu@asiaa.sinica.edu.tw
}

\begin{abstract}
We have conducted a survey of deuterium fractionation of $\mathrm{N}_{2} \mathrm{H}^{+}, R_{D}\left(\mathrm{~N}_{2} \mathrm{H}^{+}\right) \equiv$ $N\left(\mathrm{~N}_{2} \mathrm{D}^{+}\right) / N\left(\mathrm{~N}_{2} \mathrm{H}^{+}\right)$, with the Arizona Radio Observatory (ARO) Submillimeter Telescope (SMT) to assess the use of $R_{D}\left(\mathrm{~N}_{2} \mathrm{H}^{+}\right)$as an evolutionary tracer among massive protostellar/cluster cores in early stages. Our sample includes 32 dense cores in various evolutionary stages, from high-mass starless cores (HMSCs), high-mass protostellar objects (HMPOs), to ultra-compact (UC) HII regions, in infrared dark clouds (IRDCs) and high infrared extinction clouds. The results show a decreasing trend in deuterium fractionation with evolutionary stage traced by gas temperature and line width (Fig. 1). A moderate increasing trend of deuterium fractionation with the $\mathrm{CO}$ depletion factor is also found among cores in IRDCs and HMSCs. These suggest a general chemical behavior of deuterated species in low- and high-mass protostellar candidates. Upper limits to the ionization degree are also estimated to be in the range of $4 \times 10^{-8}-5 \times 10^{-6}$.
\end{abstract}

Keywords. ISM: molecules — stars: formation

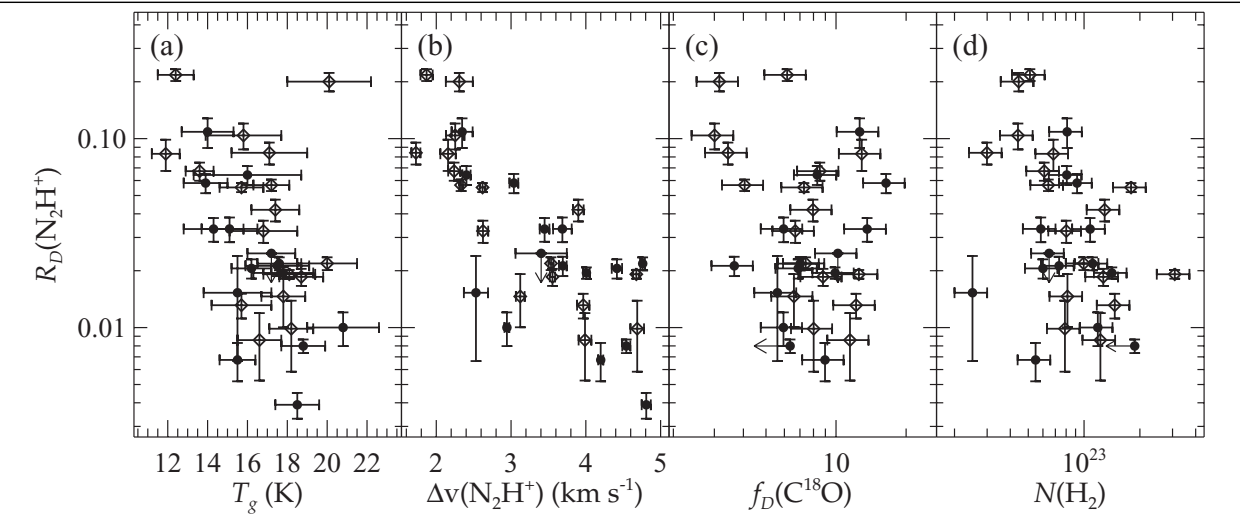

Figure 1. (a) Deuterium fractionation of $\mathrm{N}_{2} \mathrm{H}^{+}, R_{D}\left(\mathrm{~N}_{2} \mathrm{H}^{+}\right)$, vs. gas temperature, $T_{g}$, shows a decreasing trend. Cores that have been reported in Chen et al. (2011) are marked by filled circles. Recent measurements for cores in high infrared extinction clouds (Rygl et al. 2010) are marked by open diamonds. (b) $R_{D}\left(\mathrm{~N}_{2} \mathrm{H}^{+}\right)$vs. line width, $\Delta v\left(\mathrm{~N}_{2} \mathrm{H}^{+}\right)$, also shows a decreasing trend. (c) $R_{D}\left(\mathrm{~N}_{2} \mathrm{H}^{+}\right)$vs. the $\mathrm{CO}$ depletion factor, $f_{D}\left(\mathrm{C}^{18} \mathrm{O}\right)$, shows a moderate increasing trend, particularly among cores in IRDCs and HMSCs (filled circles). (d) $R_{D}\left(\mathrm{~N}_{2} \mathrm{H}^{+}\right)$vs. beam averaged column density, $N\left(\mathrm{H}_{2}\right)$, derived from the $1.2 \mathrm{~mm}$ dust continuum emission.

\section{References}

Chen, H.-R., Liu, S.-Y., Su, Y.-N., \& Wang, M.-Y. 2011, ApJ, 743, 196

Rygl, K. L. J., Wyrowski, F., Schuller, F., \& Menten, K. M. 2010, A\&A, 515, 42 